\title{
Anesthesia-related medication error: time to take action
}

\author{
Beverley A. Orser MD PhD FRCPC, Robert Byrick MD FRCPC
}

M EDICAL errors occur frequently and cause significant clinical, financial and legal costs to patients and the health care system. ${ }^{1}$ To improve patient safety, each medical and surgical discipline needs to identify the sources of error and develop evidence-based preventative strategies.

In this issue of the Journal, two reports remind us that medication errors are an ongoing challenge for patients undergoing anesthesia. Strock and colleagues describe paralysis of an awake patient resulting from the mislabelling of a pre-filled syringe that was prepared in a hospital pharmacy. ${ }^{2}$ Murdoch et al., report a near-miss incident resulting from the stocking of look-alike bottles that contain different concentrations of nitroglycerine in the labour suite. ${ }^{3}$ Indeed, a recent report by the National Health Service (NHS) of the United Kingdom entitled Building a safer NHS for patients: improving medication safety, suggests that the risk of serious drug errors is greater in anesthetic practice than other specialties. ${ }^{4}$ Recommendations in the NHS report aimed at improving drug safety in anesthetic practice are summarized in the Table.

Is there evidence to support the claim that the potential for serious drug error is highest in anesthetic practice? The incidence of medication error during anesthesia is uncertain; however, a prospective study of 55,426 procedures reported that drug errors occurred in 63 cases $(0.11 \%) .{ }^{5}$ Another study of 7,794 patients reported that the incidence of a drug administration error was $0.75 \%{ }^{6}$ Most anesthesiologists reported being involved in at least one drug error although most of the errors were inconsequential. ${ }^{7}$ The incidence of drug error is astonishingly low given the millions of drugs administered during anesthetic care. Nevertheless, the potential for harm is great, considering the potency of anesthetic drugs, the fast-pace of the operating room and multi-tasking by anesthesiologists.

Outcome studies such as analyses of closed malpractice claims are important sources of information about
TABLE Recommendations to reduce the risk of drug error in anesthesia Modified from Building a safer NHS for patients: improving medication safety. ${ }^{4}$

1. Anesthesiologists should be aware of the risks of drug errors and ensure that checking procedures are in place. Errors often occur in situations of haste, distraction or fatigue.

2. Lighting of the operating room environment is critical for safety. In situations of reduced lighting, specific arrangements should be made for checking anesthetic drugs.

3. Drug storage arrangements should be consistent in all anesthetic care delivery units.

4. Ampoules should be read and re-read before drugs are drawn up into a syringe. Errors are unlikely to be detected once the syringe is prepared.

5. Ideally, drugs are prepared by the person who will administer them, immediately before use.

6. Syringes should be labelled with the name and concentration.

7. Syringes intended for an emergency should be stored away from the immediate work area.

8. The international colour-coded syringe labelling system should be used.

9. Consider using pre-filling syringes for emergency drugs that are prepared by the pharmacy unit to assure quality of contents and accurate labelling.

10. Pharmacists should regularly visit the operating rooms to ensure safe drug use.

11. When drug manufactures, packaging and formulation changes, anesthesiologists should be alerted to the change before the drugs are provided in the operating rooms.

This list adds to previously published recommendations. NHS $=$ National Health Service.

adverse events. A recent analysis by the Canadian Medical Protective Association (CMPA) suggests that medication errors are the leading cause of adverse anesthetic-related events.

The CMPA (www.cmpa-acpm.ca) generously provided the following information in its effort to improve patient safety and reduce liability for anesthesiologists. CMPA is a medical-legal defense organization for physicians who practice in Canada. It is funded and operated 
on a 'not-for-profit' basis for physicians and by physicians. Its membership comprises $95 \%$ of the doctors licensed to practice in Canada $(>62,000)$. Thus, nearly all malpractice claims against Canadian anesthesiologists are managed by the CMPA.

An analysis of closed malpractice claims showed that medication issues are a leading cause of malpractice litigation against Canadian anesthesiologists. From 1998 to 2002 , there were 232 closed legal actions. Claims against anesthesiologists represented $3.5 \%$ of the total number of claims against all physicians $(n=6,628)$. Critical incidents that triggered the legal action were defined as omission or commission of evaluation or management that led to a problem(s). The causes of critical incidents were divided into six broad categories which are defined in the figure legend: performance, communication, diagnosis, administration, medication, and conduct (Figure). The single most common cause of malpractice action against anesthesiologists was a medication-related event. Drug issues were involved in $52 \%$ of claims; occurring in 120 patients over the five years (Figure). Notably, the number of claims involving medications far exceeded problems resulting from performance and diagnostic issues. Furthermore, the cost of medication-related events was high; nearly two thirds of all monetary damages awarded to plaintiffs were awarded for cases involving drug-related events. Thus, payment costs were disproportionate to the number of drug-related claims $(52 \%)$. A more detailed analysis of the CMPA database is not currently available but will likely be undertaken in the future. While recognizing the CMPA Closed Claims analysis does not represent overall risk, it nevertheless offers insight into causes of infrequent but potentially catastrophic events. Adverse events in the CMPA database are likely "the tip of the iceberg," representing only those cases that lead to litigation.

The CMPA data are consistent with other reports that indicate medication is the leading cause of adverse events for anesthesiologists. ${ }^{8}$ Interestingly, the incidence of drug-based cases reported in the CMPA database differs significantly in magnitude from the analysis by the ASA Closed Claims project. ${ }^{9}$ This difference is suspected to result in part from different categorization methodologies. Drug errors represented 205 (4\%) of 5,803 claims reviewed by the ASA Closed Claims project. The proportion of drug errors in the ASA database has remained relatively constant at approximately $4 \%$ for the 1980s and 1990s. The reasons for the proportion of drug-related cases in the ASA and CMPA databases (4\% vs $52 \%$ ) warrant further investigation.

What strategies can effectively reduce medicationrelated adverse events in anesthesia? Efforts to

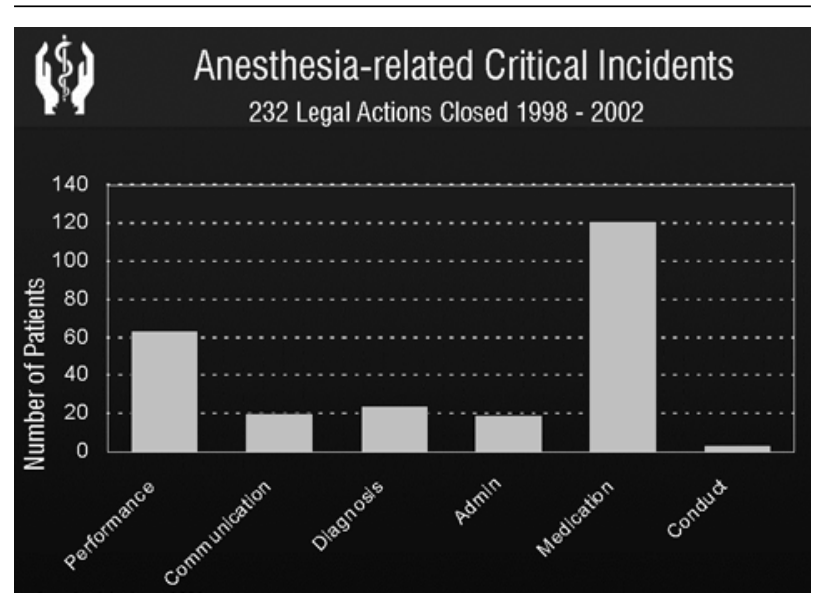

FIGURE Anesthesia-related critical incidents were categorized by the CMPA as; performance - issues regarding technical performance, wrong patient, body part, foreign body, equipment problems, delay/failure to: perform procedure or refer, monitor or attend patient; and performance of unnecessary or contra-indicated treatment; communication (comm) - communication issues with patients/family, physicians, health professionals and non-medical personnel, and alleged/proven consent issues; diagnosis - delay or misdiagnosis of initial problem(s) or complication(s) due to delay/failure to: review reports, perform diagnostic tests or refer, monitor or attend patient, misinterpretation of results, and equipment problems; administration (admin) - issues related to inadequate, illegible, or altered/falsified medical records, insufficient/unavailable resources, and administrative policies/procedures; medication - issues regarding medication administration, delay/failure to: prescribe medication or monitor/attend patient, wrong medication, dosage, and equipment problems; and conduct alleged/proven sexual impropriety, substance abuse, and inappropriate manner/unethical behaviour.

improve drug safety must be coordinated at national and international levels. To this end, the Canadian Anesthesiologists' Society (CAS) established a Patient Safety Committee that works cooperatively with the Anesthesia Patient Safety Foundation and other anesthesia organizations. The identification of adverse drug events as threats to patient safety in anesthetic practice is a major step forward. Fortunately, solutions for many drug-related problems do not require expensive, complex interventions. For example, the development of unambiguous drug labels that are easy to read and designed primarily for safety rather than marketing is an important starting point. ${ }^{10}$ The Medication Safety subcommittee of the CAS identified the three priority programs: drug labelling, infusion pump safety and medication error reporting. The CAS Safety Committee will also address 'Human Factors' that play a major role in errors including fatigue, aging, disabilities of care providers (e.g., deteriorating eyesight with age) and trained assistants as a 
second line of defense. We also require "made in Canada" solutions to address unique challenges such as bilingual drug labels. Once preventative recommendations are developed, the CAS Guidelines to the Practice of Anesthesia can be modified for widespread implementation, as in monitoring standards. The establishment of a voluntary reporting program for anesthesia-related adverse events will be another major advance. Currently, drug errors or near-misses can be reported to the Institute of Safe Medication Practices Canada (www.ismp-canada.org). Protection from litigation for the reporter must be enacted in all provinces if adverse event reporting programs are to succeed. Finally, there are many provincial and national organizations interested in patient safety. The Canadian government recently announced the formation of the Canadian Patient Safety Institute. Colleges of Physicians and Surgeons and Hospital Associations have a vested interest in preventing adverse outcomes and reducing litigation. Collaborations between national organizations such as the CMPA and CAS enhance our understanding of patient safety issues.

In the past, anesthesiology has demonstrated leadership in patient safety and anesthesia-related mortality has decreased. However, as Lagasse emphasized, there is still much work to be done and complacency is unwarranted. ${ }^{11}$ We thank the CMPA for allowing a current analysis of Canadian anesthesia-related malpractice claims to be published in a peer-reviewed journal. This information will help the specialty of anesthesiology establish medication safety as a priority for risk reduction.

\section{Les erreurs de médica- tion reliées à l'anesthésie : il est temps d'agir}

Les erreurs médicales sont fréquentes et ont un coût clinique, financier et légal important pour les patients et le système de santé. ${ }^{1}$ L'amélioration de la sécurité des patients passe par la nécessité pour toutes les disciplines médicales et chirurgicales d'identifier les sources d'erreur et de développer une prévention fondée sur des données probantes.
Dans le présent numéro du Journal, Strock et coll. décrivent la paralysie d'un patient éveillé, conséquence du mauvais étiquetage d'une seringue préremplie dans une pharmacie d'hôpital. ${ }^{2}$ Un rapport récent du National Health Service (NHS) du Royaume-Uni recommande que les pharmaciens préparent des seringues prêtes à l'emploi avec des médicaments d'urgence pour réduire les erreurs de médication reliées à l'anesthésie. ${ }^{3}$ Intitulé Building a safer NHS for patients: improving medication safety, le rapport montre que le risque d'erreurs sérieuses de médication est plus élevé en anesthésie que dans toute autre spécialité. ${ }^{4}$ D'autres recommandations, qui visent l'amélioration de la sécurité des médicaments en anesthésie, sont résumées dans le Tableau. Le cas décrit par Strock et coll. nous rappelle qu'il n'existe pas de solution simple aux erreurs de médicaments.

Qu'est-ce qui prouve que le potentiel d'erreurs sérieuses de médication est plus élevé en anesthésie ? L'incidence d'erreur de médication en anesthésie est incertaine ; toutefois, une étude prospective de $\mathbf{5 5} 426$ opérations a montré que des erreurs sont survenues dans 63 cas $(0,11 \%) .{ }^{5}$ Une autre étude de 7794 patients a rapporté une incidence de $0,75 \% .{ }^{6}$ La plupart des anesthésiologistes disent avoir été impliqués au moins une fois dans une erreur de médicament, quoique la majorité des erreurs aient été sans conséquences. ${ }^{7}$ L'incidence d'erreur est étonnamment faible, car des millions de médicaments sont administrés pendant l'anesthésie. Mais le potentiel de préjudice est grand, considérant la puissance des anesthésiques, la grande activité de la salle d'opération et les multitâches des anesthésiologistes. Des études de résultats, comme les analyses de règlement des poursuites pour faute professionnelle, renseignent beaucoup sur les effets indésirables. Une récente analyse de l'Association canadienne de protection médicale (ACPM) montre que les erreurs de médication sont la principale cause d'incidents reliés à l'anesthésie.

L'ACPM (www.cmpa-acpm.ca) a généreusement offert les informations suivantes dans le but d'améliorer la sécurité des patients et de réduire la responsabilité des anesthésiologistes. L'ACPM est un organisme de défense médico-légale pour les médecins en pratique au Canada. Il est financé et administré, sans but lucratif, pour les médecins et par les médecins. Ses membres comptent $95 \%$ de médecins admis à la pratique au Canada (> 62 000). Ainsi, presque toutes les plaintes pour faute professionnelle contre des anesthésiologistes canadiens sont traitées par l'ACPM.

Une analyse des règlements de poursuites a montré que les questions de médication sont la principale cause de litige pour faute professionnelle contre des anesthé- 
TABLEAU Recommandations pour réduire le risque d'erreur de médicament en anesthésie Adapté de Building a safer NHS for patients: improving medication safety. ${ }^{4}$

1. Les anesthésiologistes doivent connaitre les risques d'erreurs de médicaments et s'assurer que des mesures de vérification sont en place. Les erreurs surviennent souvent dans des situations de hâte, de distraction ou de fatigue.

2. L'éclairage de la salle d'opération est critique pour la sécurité. Il faut prévoir les mesures permettant de vérifier les anesthésiques quand l'éclairage est réduit.

3. Le stockage des médicaments doit être uniforme dans toutes les unités où l'anesthésie est administrée.

4. Les ampoules doivent être lues et relues avant de remplir les seringues de médicaments. Il est peu probable qu'on détecte des erreurs une fois la seringue préparée.

5. Idéalement, les médicaments sont préparés immédiatement avant l'usage par la personne qui va les administrer.

6. Les seringues doivent être étiquetées avec le nom et la concentration.

7. Les seringues prévues pour une urgence doivent être gardées à distance de l'aire de travail immédiate.

8. Il faut utiliser le système international d'étiquetage de seringues, codé par couleurs.

9. Penser à utiliser des seringues préremplies par la pharmacie pour des médicaments d'urgence afin d'assurer la qualité du contenu et la conformité de l'étiquetage.

10. Les pharmaciens doivent visiter régulièrement les salles d'opération pour vérifier la sécurité de l'usage des médicaments.

11. Lorsque la fabrication, l'emballage et la formulation des médicaments changent, les anesthésiologistes doivent être mis au courant avant que les médicaments soient apportés dans les salles d'opération.

La présente liste s'ajoute aux recommandations publiées antérieurement.

NHS = National Health Service (Service de santé national)

siologistes canadiens. De 1998 à 2002, il y a eu 232 poursuites réglées. Ces poursuites ont représenté 3,5\% de toutes les poursuites contre les médecins $(n=6$ 628). Les motifs des actions légales ont été définis comme l'omission ou la réalisation d'une évaluation ou d'un traitement qui a conduit à un, ou à des, problème(s). Les causes ont été divisées en six grandes catégories : la performance, la communication, le diagnostic, l'administration, la médication et la conduite (Figure). La seule cause fréquente de poursuite contre les anesthésiologistes était un événement relié aux médicaments. Ces derniers ont fait l'objet de $52 \%$ des poursuites et ont touché 120 patients au cours de cinq années (Figure). Le nombre de poursuites concernant la médication a dépassé de loin celui des erreurs de performance ou de diagnostic. De plus, le coût des événements liés à la médication était élevé, puisque près des deux tiers de tous les dommages monétaires payés l'ont été dans ce contexte. Le coût des règlements a

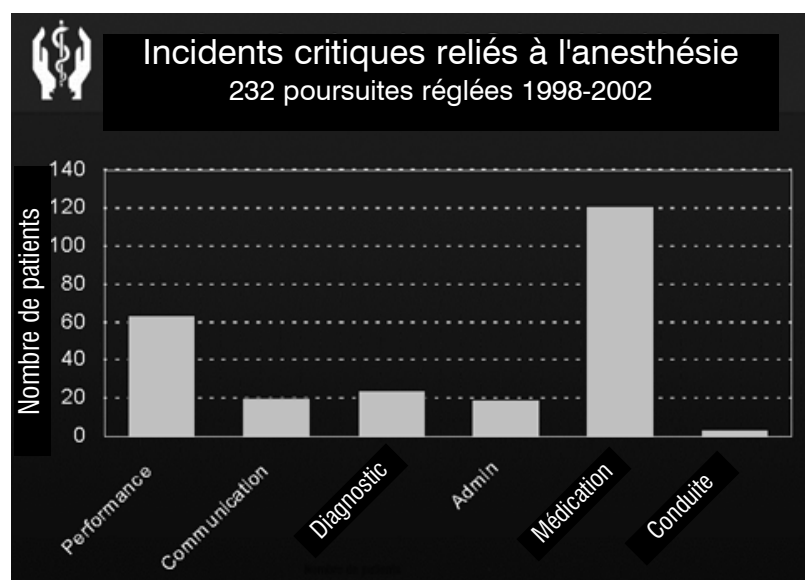

FIGURE Les incidents critiques reliés à l'anesthésie par l'ACPM sont catégorisés selon : la performance-performance technique, mauvais patient, mauvaise partie du corps, corps étranger, problèmes de matériel, retard/échec à intervenir ou à confier le patient à un collègue, à le placer sous moniteur ou à s'occuper de lui ; administration d'un traitement non nécessaire ou contre-indiqué ; la communication (comm) - communication avec les patients/la famille, les médecins, les professionnels de la santé et le personnel non médical et les questions de consentement présumé/confirmé ; le diagnostic - retard à diagnostiquer ou mauvais diagnostic du problème ou de la complication initial(e) causés par un retard/échec à : réviser les dossiers, réaliser les tests diagnostics ou diriger le patient, le surveiller ou s'en occuper, mauvaise interprétation des résultats et problèmes de matériel ; l'administration (admin) - questions reliées à des dossiers médicaux incomplets, illisibles ou altérés/falsifiés, des ressources insuffisantes/indisponibles et des politiques/procédures administratives; la médication - l'administration de la médication, un délai/échec à : prescrire la médication ou suivre/s'occuper du patient, mauvaise médication, mauvais dosage et problèmes de matériel ; la conduite - comportement sexuel inconvenant présumé/confirmé, pharmacodépendance et manières incorrectes/comportement non éthique.

donc été disproportionné au nombre de poursuites (52 $\%)$. Actuellement, il n'y a pas d'analyse plus détaillée des données de l'ACPM, mais elle sera probablement réalisée à l'avenir. L'analyse des règlements de poursuites de l'ACPM ne représente certainement pas le risque global ; elle offre néanmoins un aperçu des cas d'événements rares mais possiblement catastrophiques. Les événements indésirables de la base de données de l'ACPM ne sont sans doute que la "pointe de l'iceberg», puisqu'ils ne représentent que les cas de litige.

Les données de l'ACPM correspondent à celles d'autres rapports indiquant la médication comme principale cause d'événements indésirables en anesthésie. ${ }^{8}$ Il est intéressant de noter que l'incidence de cas liés aux médicaments rapportés dans la base de 
données de l'ACPM diffère significativement de l'analyse faite par l'ASA Closed Claims project.' Cette différence pourrait résulter en partie de méthodologies de catégorisation différentes. Les erreurs de médicament représentent 205 (4\%) des 5803 réclamations revues par l'ASA Closed Claims project. Ce pourcentage est demeuré relativement constant à $4 \%$ environ pour les années 1980 et 1990. L'explication des différences de données entre l'ASA et l'ACPM (4 $\%$ vs $52 \%$ ) mérite une investigation plus poussée.

Quelles stratégies peuvent efficacement réduire les événements indésirables liés aux anesthésiques ? Il faut certainement des efforts nationaux et internationaux concertés.

À cette fin, la Société canadienne des anesthésiologistes (SCA) a mis sur pied un Comité sur la sécurité des patients en collaboration avec l'Anesthesia Patient Safety Foundation et d'autres organismes d'anesthésie. Reconnaitre le problème est un important pas en avant. Heureusement, la solution n'exige pas toujours des interventions coûteuses et complexes. Par exemple, le développement d'étiquettes claires, faciles à lire et conçues d'abord pour la sécurité plutôt que pour la commercialisation, est un bon point de départ. ${ }^{10}$ Le sous-comité sur la sécurité des médicaments de la SCA a défini trois priorités : l'étiquetage des médicaments, la sécurité des pompes à perfusion et la notification des erreurs de médication. Le Comité sur la sécurité de la SCA s'intéressera aussi aux «facteurs humains», comme la fatigue, l'âge, l'inaptitude du personnel soignant (par ex., la diminution de la vue avec l'âge) et les assistants formés comme seconde ligne de défense. Aussi, des solutions «fabriquées au Canada» résoudraient des problèmes particuliers comme le bilinguisme de l'étiquetage. Une fois élaborées les recommandations préventives, on peut modifier le Guide canadien d'exercice de l'anesthésie pour une application généralisée, comme dans le cas du monitorage. L'élaboration d'un programme de notification volontaire des événements indésirables sera un autre progrès majeur. Actuellement, les erreurs de médicaments ou les manquements évités de justesse peuvent être rapportés à l'Institute of Safe Medication Practices-Canada (www.ismp-canada.org). Le succès de la notification passe par l'adoption dans toutes les provinces d'une loi protégeant l'informateur contre les litiges. Finalement, le gouvernement canadien a récemment annoncé la formation d'une Fondation nationale sur la sécurité des patients, sujet qui intéresse de nombreux organismes provinciaux et nationaux comme les Collèges de médecins et chirurgiens et les Associations d'hôpitaux. La collaboration entre l'ACPM et la SCA accroît notre compréhension des questions relatives à la sécurité des patients.
Dans le passé, l'anesthésiologie a fait preuve de leadership dans la question de la sécurité des patients et la mortalité reliée à l'anesthésie a diminué. Cependant, comme Lagasse le souligne, il y a encore beaucoup à faire et le contentement de soi est injustifié. ${ }^{11}$ Nous remercions l'ACPM de nous avoir permis, pour la première fois, de publier une analyse des poursuites pour faute professionnelle reliée à l'anesthésie dans un journal scientifique. Cette information servira l'anesthésiologie qui veut faire de la sécurité de la médication une priorité dans la réduction du risque.

\section{References}

1 Brennan TA, Leape LL, Laird NM, et al. Incidence of adverse events and negligence in hospitalized patients: results of the Harvard Medical Practice Study I. 1991. Qual Saf Health Care 2004; 13: 145-51.

2 Strock D, Kuczkowski KM, Greenberg M. Accidental administration of succinylcholine for the treatment of hypotension in a labouring parturient (Letter). Can J Anesth 2004; 853-54.

3 Murdoch JA, Lane J, Goldstein DH. Drug labelling and a near miss in the labour suite (Letter). Can J Anesth 2004; 51: 854-55.

4 Building a safer NHS for patients: improving medication safety. A report by the Chief Pharmaceutical Officer. Jim Smith (Ed.). 2004, Copyright holder: The Crown. Available from URL;

http://www.dh.gov.uk/assetRoot/04/07/15/07/04 071507.pdf.

5 Fasting S, Gisvold SE. Adverse drug errors in anesthesia, and the impact of coloured syringe labels. Can J Anesth 2000; 47: 1060-7.

6 Webster CS, Merry AF, Larsson L, McGrath KA, Weller $J$. The frequency and nature of drug administration error during anaesthesia. Anaesth Intensive Care 2001; 29: 494-500.

7 Orser BA, Chen RJ, Yee DA. Medication errors in anesthetic practice: a survey of 687 practitioners. Can J Anesth $2001 ; 48$ : 139-46.

8 Cooper JB, Newbower RS, Kitz RJ. An analysis of major errors and equipment failures in anesthesia management: considerations for prevention and detection. Anesthesiology 1984; 60: 34-42.

9 Bowdle TA. Drug administration errors from the ASA Closed Claims Project. ASA Newsletter 2003; 67: 11-3.

10 Chen R, Orser BA. Medication labels: for whose benefit? [(Letter) published erratum appears in Can J Anaesth 1996 43: 319]. Can J Anaesth 1996; 43: 194-5.

11 Lagasse RS. Anesthesia safety: model or myth? A review of the published literature and analysis of current original data. Anesthesiology 2002; 97: 1609-17. 